\title{
DUALITIES OF DIFFERENTIAL GEOMETRIC INVARIANTS ON CUSPIDAL EDGES ON FLAT FRONTS IN THE HYPERBOLIC SPACE AND THE DE SITTER SPACE
}

\author{
KENTARO SAJI AND KEISUKE TERAMOTO
}

\begin{abstract}
We compute the differential geometric invariants of cuspidal edges on flat surfaces in hyperbolic 3-space and in de Sitter space. Several dualities of invariants are pointed out.
\end{abstract}

\section{INTRODUCTION}

In [6], one Weierstrass type representation formula for flat surfaces in the hyperbolic 3-space $H^{3}$ is obtained, and two representations are given in 21]. In [21, a notion flat fronts for flat surfaces which admit certain singularities is defined and the Weierstrass type representation formula is extended to the representation formula for flat fronts. This formula represents flat fronts via two holomorphic functions (called the Weierstrass data). In 20, criteria for cuspidal edges and swallowtails are given in terms of the Weierstrass data, and it is shown that these singularities are generic singularities of flat fronts in $H^{3}$. For a flat fronts in $H^{3}$, it is known that its unit normal vector taking the value in the de Sitter 3-space $S_{1}^{3}$ (called the de Sitter Gauss map) is also a flat front in $S_{1}^{3}$, and it is constructed from the same data. It is known that the sets of singular points of these two flat fronts coincide. Flat fronts are special cases of linear Weingarten fronts. In 22, a global Weierstrass type representation of linear Weingarten fronts is given, and global properties of them are studied and correspondence between dual surfaces of them are given (see [22] for detail, see also [7,17]).

One can regard that these two flat surfaces are in a dual relation. It is introduced in [11, that a formulation considering this duality as a double Legendrian fibration in contact geometry. See 13, 14 for studies of inear Weingarten surfaces from this viewpoint. On the other hand, a front is a surface in a 3-space with well-defined unit normal vector even on the set of singular points. Since there is a unit normal vector, fronts can be studied from the differential geometric viewpoint. Many geometric invariants of cuspidal edges and other singular points are introduced and geometric properties of them are investigated. (see $[2,5,2,10,15,25,29,33,35$ for example). Since flat fronts are determined by the Weierstrass data, it is natural to consider a relation between the data and the invariants of singular points. This relation can be regarded as geometric meanings of the data on singular points.

In this paper, we give explicit expressions for invariants of cuspidal edges in terms of the Weierstrass data $(\alpha, \beta)$ (Theorem 3.4). We can observe several dualities of

2010 Mathematics Subject Classification. Primary 53A55; Secondary 53A35, 57R45.

Key words and phrases. Cuspidal edge, flat front, duality.

Partly supported by the JSPS KAKENHI Grant Number 18K03301 and the JSPS KAKENHI Grant Number 17J02151. 
geometric invariants and singularities of cuspidal edges on flat fronts in $H^{3}$ and $S_{1}^{3}$. Using such expressions, we characterize the condition that the set of singular points which consists of cuspidal edges is a line of curvature (Proposition 4.1). Furthermore, we show a relation between lines of curvature and cone-like singular points (Corollary 4.3). For geometric meanings of cone-like singularities of flat fronts in $H^{3}$, see $[8,20,21]$.

\section{Preliminaries}

2.1. Fronts in $H^{3}$ and $S_{1}^{3}$. Let $\boldsymbol{R}_{1}^{4}$ be the Lorentz-Minkowski 4-space with the inner product $\langle\rangle=,(-+++)$. Let

$$
\begin{aligned}
H^{3} & =\left\{x=\left(x_{0}, x_{1}, x_{2}, x_{3}\right) \in \boldsymbol{R}_{1}^{4} \mid\langle x, x\rangle=-1, x_{0}>0\right\}, \\
S_{1}^{3} & =\left\{x \in \boldsymbol{R}_{1}^{4} \mid\langle x, x\rangle=1\right\}
\end{aligned}
$$

be the hyperbolic and the de Sitter 3-spaces. Although dual relations of surfaces in these two pseudo-spheres has been known (see [18, (2.3)], [19, (1.9)] for example), we use the formulation in [11] to working on Legendrian dualities. Following [11, we set $\Delta_{1} \subset H^{3} \times S_{1}^{3}$ by

$$
\Delta_{1}=\left\{(x, y) \in H^{3} \times S_{1}^{3} \mid\langle x, y\rangle=0\right\},
$$

and set $\pi_{1}: \Delta_{1} \rightarrow H^{3}, \pi_{2}: \Delta_{1} \rightarrow S_{1}^{3}$ by

$$
\pi_{1}(x, y)=x, \quad \pi_{2}(x, y)=y .
$$

Moreover, we set two 1-forms

$$
\theta_{1}=-x_{0} d y_{0}+\left.\sum_{i=1}^{3} x_{i} d y_{i}\right|_{\Delta_{1}}, \quad \theta_{2}=-y_{0} d x_{0}+\left.\sum_{i=1}^{3} y_{i} d x_{i}\right|_{\Delta_{1}}
$$

Then $\theta_{1}^{-1}(0)$ and $\theta_{2}^{-1}(0)$ determine the same tangent plane field over $\Delta_{1}$ which is denoted by $K$. It is well-known that the pair $\left(\Delta_{1}, K\right)$ is a contact manifold and $\pi_{1}$ and $\pi_{2}$ are Legendrian fibrations ([11, Theorem 2.2]). This formulation is introduced for investigating surfaces in the lightcones. See [11] for other dualities among pseudo-spheres. It should be remarked that dualities of hypersurfaces in pseudo-spheres in the Lorentz-Minkowski space are also found independently (see [3, 23, 24]).

Let $\left(N, d s^{2}\right)$ be a Riemannian or a semi-Riemannian 3-manifold. Let $U \subset \boldsymbol{R}^{2}$ be a domain. A map $k: U \rightarrow N$ is a frontal if there exists a map $L: U \rightarrow T_{1} N$ such that $\pi \circ L=k$ and $d s^{2}(d k(X), L(p))=0$, where $\pi: T_{1} N \rightarrow N$ is the unit tangent bundle. The lift $L$ is called an isotropic lift of $k$. A frontal $k$ is a front if an isotropic lift can be taken as an immersion. Since the unit tangent bundle of $H^{3}$ can be identified with $H^{3} \times S_{1}^{3}$, we can rewrite this setting by using $\Delta_{1}$. A map $L=(f, g): U \rightarrow \Delta_{1}$ is isotropic if $L^{*} \theta_{1}=0$. A map $f: U \rightarrow H^{3}$ (respectively, $\left.g: U \rightarrow S_{1}^{3}\right)$ is a frontal if there exists a map $g: U \rightarrow S_{1}^{3}$ (respectively, $f: U \rightarrow H^{3}$ ) such that the pair $(f, g): U \rightarrow \Delta_{1}$ is isotropic. A frontal $f: U \rightarrow H^{3}$ (respectively, $\left.g: U \rightarrow S_{1}^{3}\right)$ is a front if the map $L=(f, g)$ can be taken as an immersion. If $(f, g): U \rightarrow \Delta_{1}$ is isotropic, then we say that $f$ and $g$ are $\Delta_{1}$-dual each other, $g$ is a $\Delta_{1}$-dual of $f$, and $f$ is a $\Delta_{1}$-dual of $g$. 
2.2. Matrix representation of $H^{3}$ and $S_{1}^{3}$. Let $\operatorname{Herm}(2)$ be the set of $2 \times 2$ Hermitian matrices. We take elements $e_{0}, e_{1}, e_{2}, e_{3} \in \operatorname{Herm}(2)$ as

$$
e_{0}=\left(\begin{array}{ll}
1 & 0 \\
0 & 1
\end{array}\right), \quad e_{1}=\left(\begin{array}{ll}
0 & 1 \\
1 & 0
\end{array}\right), \quad e_{2}=\left(\begin{array}{cc}
0 & i \\
-i & 0
\end{array}\right), \quad e_{3}=\left(\begin{array}{cc}
1 & 0 \\
0 & -1
\end{array}\right),
$$

where $i=\sqrt{-1}$. Then we have an identification $\iota: \boldsymbol{R}^{4} \rightarrow \operatorname{Herm}(2)$

$$
\iota(x)=\sum_{k=0}^{3} x_{k} e_{k}=\left(\begin{array}{cc}
x_{0}+x_{3} & x_{1}+i x_{2} \\
x_{1}-i x_{2} & x_{0}-x_{3}
\end{array}\right),
$$

where $x=\left(x_{0}, x_{1}, x_{2}, x_{3}\right)$ with the metric

$$
\langle X, Y\rangle=-\frac{1}{2} \operatorname{trace}\left(X e_{2} Y e_{2}\right),
$$

$X, Y \in \operatorname{Herm}(2)$. In particular,

$$
\langle X, X\rangle=-\operatorname{det} X \quad(X \in \operatorname{Herm}(2)) .
$$

By this identification, $H^{3}$ and $S_{1}^{3}$ are rewritten as

$$
\begin{aligned}
H^{3} & =\{X \in \operatorname{Herm}(2) \mid \operatorname{det} X=1, \operatorname{trace} X>0\}=\left\{A A^{*} \mid A \in S L(2, \boldsymbol{C})\right\}, \\
S_{1}^{3} & =\{X \in \operatorname{Herm}(2) \mid \operatorname{det} X=-1\}=\left\{A e_{3} A^{*} \mid A \in S L(2, \boldsymbol{C})\right\} .
\end{aligned}
$$

Furthermore, the exterior product in $T_{p} H^{3}$ and $T_{p} S_{1}^{3}$ are rewritten as

$$
X \times Y=\frac{i}{2}\left(X p^{-1} Y-Y p^{-1} X\right)
$$

for $X, Y \in T_{p} H^{3}$, or $X, Y \in T_{p} S_{1}^{3}$.

2.3. Singularities of fronts and their differential geometric invariants. Let $\left(N, d s^{2}\right)$ be a Riemannian or a semi-Riemannian 3-manifold. Let $U \subset \boldsymbol{R}^{2}$ be a domain, and $k: U \rightarrow N$ a front. For $p \in U$, considering $L(p)=(p, \nu(p)) \in T_{p}^{1} N$, we consider the signed area density function

$$
\Omega\left(k_{u}(u, v), k_{v}(u, v), \nu(u, v)\right)
$$

for a coordinate system $(u, v)$, and ()$_{u}=\partial / \partial u,()_{v}=\partial / \partial v$, where $\Omega$ is the volume form. A function $\lambda: U \rightarrow \boldsymbol{R}$ is called an identifier of singularity if it is a non-zero functional multiplication of $\Omega\left(k_{u}(u, v), k_{v}(u, v), \nu(u, v)\right)$. A singular point $p$ of $k$ is called non-degenerate if $d \lambda(p) \neq 0$, where $\lambda$ is an identifier of singularity.

Let $p \in U$ be a non-degenerate singular point of a front $k: U \rightarrow N$. Then by the non-degeneracy of $p$, the set of singular points $\Sigma(k)$ is a regular curve near $p$, and hence we take a parameterization $\gamma(t)(\gamma(0)=p)$ of it. We call $\gamma$ a singular curve. We set $\hat{\gamma}=k \circ \gamma$. One can show that there exists a vector field $\eta$ on a sufficient small neighborhood of $p$ such that if $p \in \Sigma(k)$, then $\operatorname{ker} d k_{p}=\left\langle\eta_{p}\right\rangle$. We call $\eta$ the null vector field. The restriction of $\eta$ on $\Sigma(k)$ can be parameterized by the parameter $t$ of $\gamma$. We denote by $\eta(t)$ the null vector field along $\gamma$.

Definition 2.1. Let $\boldsymbol{R}^{3}$ be the Euclidean 3-space and $k:\left(\boldsymbol{R}^{2}, 0\right) \rightarrow\left(\boldsymbol{R}^{3}, 0\right)$ a $C^{\infty}$ map-germ. The map-germ $k$ at 0 is a cuspidal edge (respectively, a swallowtail) if is is $\mathcal{A}$-equivalent to the map-germ $(u, v) \mapsto\left(u, v^{2}, v^{3}\right)$ (respectively, $(u, v) \mapsto$ $\left.\left(u, 4 v^{3}+2 u v, 3 v^{4}+u v^{2}\right)\right)$ at the origin. Here, two map-germs $g_{1}, g_{2}:\left(\boldsymbol{R}^{2}, 0\right) \rightarrow$ $\left(\boldsymbol{R}^{3}, 0\right)$ are $\mathcal{A}$-equivalent if there exist diffeomorphism-germs $\Xi_{s}:\left(\boldsymbol{R}^{2}, 0\right) \rightarrow\left(\boldsymbol{R}^{2}, 0\right)$ and $\Xi_{t}:\left(\boldsymbol{R}^{3}, 0\right) \rightarrow\left(\boldsymbol{R}^{3}, 0\right)$ satisfying $g_{2} \circ \Xi_{s}=\Xi_{t} \circ g_{1}$. 
If $k:\left(\boldsymbol{R}^{2}, 0\right) \rightarrow\left(\boldsymbol{R}^{3}, 0\right)$ is a cuspidal edge or a swallow, then it is a front and 0 is a non-degenerate singular point. There are useful criteria for them. Let $k: U \rightarrow \boldsymbol{R}^{3}$ be a front and $p \in U$ a non-degenerate singular point. Let $\gamma(t)$ be a parametrization $(\gamma(0)=p)$ of the singular curve, and $\eta(t)$ a null vector field. We set $\delta(t)=\operatorname{det}\left(\gamma^{\prime}(t), \eta(t)\right)$. For a non-degenerate singular point $p$ of $k$, the map-germ $k$ at $p$ is cuspidal edge (respectively, swallowtail) if and only if $\delta(0) \neq 0$ (respectively, $\left.\delta(0)=0, \delta^{\prime}(0)=0\right)$. See [20, Proposition 1.3].

Let $M$ be $H^{3}$ or $S_{1}^{3}$. Let $f: U \rightarrow M$ be a front and let $f$ at $p$ be a cuspidal edge, and $g$ a $\Delta_{1}$-dual of $f$. A pair of vector fields $(\xi, \eta)$ is called adapted if $\xi$ is tangent to the singular set and $\eta$ is a null vector field of $f$. By the criterion for cuspidal edge, $\eta \lambda \neq 0$ holds. Thus $\xi f$ and $\nabla_{\eta} \eta f$ are linearly independent, in particular, $\xi f \neq 0$ and $\xi f \times \nabla_{\eta} \eta f \neq 0$ hold. We define

$$
\begin{gathered}
\kappa_{s}(t)=\left.\operatorname{sgn}\left(\lambda_{\eta}\right) \frac{\Omega\left(\xi f, \nabla_{\xi}(\xi f), g\right)}{|\xi f|^{3}}\right|_{(u, v)=\gamma(t)}, \quad \kappa_{n}(t)=\left.\frac{\left\langle\nabla_{\xi}(\xi f), g\right\rangle}{|\xi f|^{2}}\right|_{(u, v)=\gamma(t)}, \\
\kappa_{t}(t)=\frac{\Omega\left(\xi f, \nabla_{\eta}(\eta f), \nabla_{\xi} \nabla_{\eta}(\eta f)\right)}{\left|\xi f \times \nabla_{\eta}(\eta f)\right|^{2}} \\
\quad-\left.\frac{\Omega\left(\xi f, \nabla_{\eta}(\eta f), \nabla_{\xi}(\xi f)\right)\left\langle\xi f, \nabla_{\eta}(\eta f)\right\rangle}{|\xi f|^{2}\left|\xi f \times \nabla_{\eta}(\eta f)\right|^{2}}\right|_{(u, v)=\gamma(t)}, \\
\kappa_{c}(t)=\left.\frac{|\xi f|^{3 / 2} \Omega\left(\xi f, \nabla_{\eta}(\eta f), \nabla_{\eta} \nabla_{\eta}(\eta f)\right)}{\left|\xi f \times \nabla_{\eta}(\eta f)\right|^{5 / 2}}\right|_{(u, v)=\gamma(t)},
\end{gathered}
$$

where $\gamma(t)$ is a parametrization of $\Sigma(f), \lambda$ is an identifier of singularity, and $\Omega$ is the volume form and under the identification $T_{p} \boldsymbol{R}_{1}^{4}=\boldsymbol{R}_{1}^{4}$, it can be calculated by $\Omega(X, Y, Z)=\langle X \times Y, Z\rangle$ for $X, Y, Z \in T_{f(p)} H^{3}$ or for $X, Y, Z \in T_{f(p)} S_{1}^{3}$. Here, $\nabla$ is the metric connection defined for a vector $\zeta \in T_{f(p)} H^{3}$,

$$
\nabla_{\zeta} k=\zeta k+\langle\zeta k, f\rangle f \in T_{f(p)} H^{3}
$$

and for a vector $\zeta \in T_{f(p)} S_{1}^{3}$,

$$
\nabla_{\zeta} k=\zeta k-\langle\zeta k, g\rangle g \in T_{f(p)} S_{1}^{3} .
$$

The above functions $\kappa_{s}, \kappa_{n}, \kappa_{t}$ and $\kappa_{c}$ do not depend on the choices of the parameters of $\Sigma(f), \Sigma(g)$ nor on the choice of $(\xi, \eta)$, and they are called singular curvature, limiting normal curvature, cusp-directional torsion (cuspidal torsion) and cuspidal curvature respectively. See [29] (for $\kappa_{s}$ and $\kappa_{n}$ ), 26] (for $\left.\kappa_{t}\right)$ and [27] (for $\left.\kappa_{c}\right)$ for detail.

\section{Flat Fronts in $H^{3}$ AND $S_{1}^{3}$}

Let $U \subset \boldsymbol{C}$ be a domain, and $\alpha, \beta: U \rightarrow C \backslash\{0\}$ holomorphic functions. We consider a solution $A: U \rightarrow S L(2, C)$ of the differential equation

$$
A^{\prime}(z)=A(z) D(z) \quad\left(D(z)=\left(\begin{array}{cc}
0 & \alpha(z) \\
\beta(z) & 0
\end{array}\right)\right), \quad{ }^{\prime}=\frac{d}{d z} .
$$

Then one can see that $\operatorname{det} A$ is a constant, we take a solution satisfying $\operatorname{det} A=1$. We define maps $f: U \rightarrow H^{3}$ and $g: U \rightarrow S_{1}^{3}$ by

$$
f(z)=A(z) A^{*}(z), \quad g(z)=A(z) e_{3} A^{*}(z) .
$$


Then it is known that $f$ is (zero intrinsic curvature) and $g$ is spacelike flat on their regular point sets [21, Proposition 2.5]. Furthermore, $f$ and $g$ are $\Delta_{1}$-dual each other.

By a calculation, we have

$$
f^{\prime}=A D A^{*}, \quad f_{\bar{z}}=A D^{*} A^{*}, \quad g^{\prime}=A D e_{3} A^{*}, \quad g_{\bar{z}}=A e_{3} D^{*} A^{*} .
$$

Thus by

$$
f^{\prime} \times f_{\bar{z}}=\frac{i}{2} \lambda A e_{3} A^{*}, \quad g^{\prime} \times g_{\bar{z}}=-\frac{i}{2} \lambda A A^{*},
$$

the singular sets $\Sigma(f)$ and $\Sigma(g)$ are coincide, where $\lambda$ is defined by

$$
\lambda=\alpha \bar{\alpha}-\beta \bar{\beta} .
$$

We see that $\lambda$ is an identifier of singularity for each $f$ and $g$. It is also known that the set of singular points of flat front in the 3 -sphere $S^{3}$ and that of its dual coincide. In [16], a characterization of the flat torus in $S^{3}$ is obtained.

3.1. Criteria for singularities of $f, g$. We give conditions for cuspidal edges and swallowtails appearing on the flat fronts in $H^{3}$ and in $S_{1}^{3}$ by the Weierstrass data $(\alpha, \beta)$. The case of the flat fronts in $H^{3}$, it is obtained in [20, Theorem 1.1], and the case of the flat fronts in $S_{1}^{3}$, the similar conditions are obtained in [4, Proposition $6]$. We shall state here conditions for cuspidal edges and swallowtails on flat fronts in $S_{1}^{3}$ in terms of the Weierstrass data $(\alpha, \beta)$.

Let $f: U \rightarrow H^{3}$ and $g: U \rightarrow S_{1}^{3}$ be flat fronts constructed by the pair $(\alpha, \beta)$ of holomorphic functions as in (3.2). Let $p$ be a non-degenerate singular point of $f$ or $g$, and $\gamma(t)$ a parametrization of singular curve near $p$. The tangent vector $\dot{\gamma}(t)=(d / d t) \gamma(t)$ to $\gamma$ can be expressed by

$$
\xi(t)=-i \lambda_{\bar{z}}(\gamma(t))=-i\left(\alpha \bar{\alpha}_{\bar{z}}-\beta \bar{\beta}_{\bar{z}}\right)(\gamma(t)) \partial_{z}+i\left(\alpha^{\prime} \bar{\alpha}-\beta^{\prime} \bar{\beta}\right)(\gamma(t)) \partial_{\bar{z}}
$$

(see [20]) under the identification $T_{p} U$ with $\boldsymbol{R}^{2}$ and $\boldsymbol{C}$ via the correspondence

$$
\zeta=a+b i \in \boldsymbol{C} \leftrightarrow(a, b) \in \boldsymbol{R}^{2} \leftrightarrow a \partial_{u}+b \partial_{v}=\zeta \partial_{z}+\bar{\zeta} \partial_{\bar{z}},
$$

where $z=u+i v$. On the other hand, following [20, p322], one can take null vector fields as follows:

Lemma 3.1. The vector field $\eta_{h}$ gives a null vector field of $f$ and $\eta_{g}$ gives a null vector field of $g$, where

$$
\eta_{h}=\frac{i}{\sqrt{\alpha \beta}}=\frac{i}{\sqrt{\alpha \beta}} \partial_{z}-\frac{i}{\sqrt{\bar{\alpha} \bar{\beta}}} \partial_{\bar{z}}, \quad \eta_{d}=\frac{1}{\sqrt{\alpha \beta}}=\frac{1}{\sqrt{\alpha \beta}} \partial_{z}+\frac{1}{\sqrt{\bar{\alpha} \bar{\beta}}} \partial_{\bar{z}} .
$$

Proof. See [20, p322] for $\eta_{h}$. The directional derivative $\eta_{d} g$ of $g$ in the direction of $\eta_{d}$ can be calculated as

$$
\eta_{d} g=A\left(\begin{array}{cc}
0 & -\frac{\alpha}{\sqrt{\alpha \beta}}+\frac{\bar{\beta}}{\sqrt{\bar{\alpha} \bar{\beta}}} \\
\frac{\beta}{\sqrt{\alpha \beta}}-\frac{\bar{\alpha}}{\sqrt{\bar{\alpha} \bar{\beta}}} & 0
\end{array}\right) A^{*} .
$$

Since $\alpha \bar{\alpha}-\beta \bar{\beta}=0$ on $\Sigma(g)$, it follows that $\eta_{d} g=0$ on $\Sigma(g)$. 
We set

$$
\begin{array}{r}
C_{h}=\operatorname{Re}\left(\frac{i \lambda^{\prime}}{\sqrt{\alpha \beta}}\right)=\frac{\operatorname{Re}\left(i \sqrt{\alpha \beta} \lambda_{\bar{z}}\right)}{|\alpha|^{2}} \\
=-\operatorname{Im}\left(\frac{\lambda^{\prime}}{\sqrt{\alpha \beta}}\right)=\operatorname{Im}\left(\frac{\lambda_{\bar{z}}}{\sqrt{\overline{\alpha \beta}}}\right)=\frac{\operatorname{Im}\left(\sqrt{\alpha \beta} \lambda_{\bar{z}}\right)}{|\alpha|^{2}}, \\
C_{d}=\operatorname{Re}\left(\frac{\lambda^{\prime}}{\sqrt{\alpha \beta}}\right)=\frac{\operatorname{Re}\left(\sqrt{\alpha \beta} \lambda_{\bar{z}}\right)}{|\alpha|^{2}} \\
=\operatorname{Im}\left(\frac{i \lambda^{\prime}}{\sqrt{\alpha \beta}}\right)=\operatorname{Im}\left(\frac{i \lambda_{\bar{z}}}{\sqrt{\bar{\alpha} \bar{\beta}}}\right)=\frac{\operatorname{Im}\left(i \sqrt{\alpha \beta} \lambda_{\bar{z}}\right)}{|\alpha|^{2}} .
\end{array}
$$

Then we have the following proposition.

Proposition 3.2. (I)([20, Theorem 1.1]) Let $f: U \rightarrow H^{3}$ be a flat front constructed by the data $(\alpha, \beta)$ as in (3.2). Let $p$ be a non-degenerate singular point of $f$. Then

(1) $f$ at $p$ is a cuspidal edge if and only if $C_{h} \neq 0$ at $p$.

(2) $f$ at $p$ is a swallowtail if and only if $C_{h}=0$ and

$$
\operatorname{Re}\left(\frac{S(\alpha)-S(\beta)}{\alpha \beta}\right) \neq 0
$$

at $p$, where $S(\alpha)$ is the Schwarzian derivative of the primitive function of $\alpha$ with respect to $z$ :

$$
S(\alpha)=\left(\frac{\alpha^{\prime}}{\alpha}\right)^{\prime}-\frac{1}{2}\left(\frac{\alpha^{\prime}}{\alpha}\right)^{2} .
$$

(II) Let $g: U \rightarrow S_{1}^{3}$ be a flat front constructed by the data $(\alpha, \beta)$ as in (3.2). Let $p$ be a non-degenerate singular point of $g$. Then

(1) $g$ at $p$ is a cuspidal edge if and only if $C_{d} \neq 0$ at $p$.

(2) $g$ at $p$ is a swallowtail if and only if $C_{d}=0$ and (3.9) at $p$.

Proof. See 20, Theorem 1.1] for the proof of (I). Since

$$
\left\{z \mid\left(\eta_{h} f, \eta_{d} f\right)=(0,0)\right\}=\left\{z \mid\left(\eta_{h} g, \eta_{d} g\right)=(0,0)\right\}=\emptyset,
$$

and $f$ and $g$ are dual each other, $f$ and $g$ are fronts. By [20, Proposition 1.3], it is enough to show that the condition (II), (1) in the proposition is equivalent to $\delta_{d}(0) \neq 0$, where $\delta_{d}(t)=\operatorname{det}\left(\xi(t), \eta_{d}(t)\right)$. By

$$
\begin{aligned}
\delta_{d}(t) & =-i\left(\frac{\lambda_{\bar{z}}}{\sqrt{\bar{\alpha} \bar{\beta}}}+\frac{\lambda^{\prime}}{\sqrt{\alpha \beta}}\right)(\gamma(t))=-2 i \operatorname{Re}\left(\frac{\lambda_{\bar{z}}}{\sqrt{\bar{\alpha} \bar{\beta}}}\right)(\gamma(t)) \\
& =\frac{-2 i \operatorname{Re}\left(\sqrt{\alpha \beta} \lambda_{\bar{z}}\right)(\gamma(t))}{|\alpha(\gamma(t))|^{2}}
\end{aligned}
$$

and the relation $\bar{\alpha} \bar{\beta}=|\alpha|^{4} /(\alpha \beta)$ holds along $\gamma$, the first assertion holds.

Next we show the second assertion of (II). We note that

$$
\lambda^{\prime}=|\alpha|^{2}\left(\frac{\alpha^{\prime}}{\alpha}-\frac{\beta^{\prime}}{\beta}\right)
$$

on $\gamma$. Thus by (3.10), $\delta_{d}$ is proportional to

$$
\tilde{\delta}_{d}=\operatorname{Re}\left(\frac{1}{\sqrt{\alpha \beta}}\left(\frac{\alpha^{\prime}}{\alpha}-\frac{\beta^{\prime}}{\beta}\right)\right) .
$$


We assume that $\delta_{d}(0)=0$, namely, $\tilde{\delta}_{d}(0)=0$. Then since

$$
\begin{aligned}
& \frac{d}{d t}\left(\left(\frac{1}{\sqrt{\alpha \beta}}\left(\frac{\alpha^{\prime}}{\alpha}-\frac{\beta^{\prime}}{\beta}\right)\right)(\gamma(t))\right) \\
& =\left\{\left(\left(\frac{\alpha^{\prime}}{\alpha}\right)^{\prime}-\left(\frac{\beta^{\prime}}{\beta}\right)^{\prime}\right) \frac{1}{\sqrt{\alpha \beta}}-\frac{1}{2}\left(\frac{\alpha^{\prime}}{\alpha}-\frac{\beta^{\prime}}{\beta}\right)\left(\frac{\alpha^{\prime}}{\alpha}+\frac{\beta^{\prime}}{\beta}\right) \frac{1}{\sqrt{\alpha \beta}}\right\} \dot{\gamma} \\
& =\frac{\dot{\gamma}}{\sqrt{\alpha \beta}}(S(\alpha)-S(\beta)) .
\end{aligned}
$$

Thus we have

$$
\frac{d}{d t}\left(\tilde{\delta}_{d}(\gamma(t))\right)=\frac{1}{2}\left(\frac{\dot{\gamma}}{\sqrt{\alpha \beta}}(S(\alpha)-S(\beta))+\overline{\left(\frac{\dot{\gamma}}{\sqrt{\alpha \beta}}(S(\alpha)-S(\beta))\right)}\right) .
$$

Here it holds that

$$
\begin{aligned}
\frac{\dot{\gamma}}{\sqrt{\alpha \beta}} & =\frac{-i|\alpha|^{2}}{\sqrt{\alpha \beta}} \overline{\left(\frac{\alpha^{\prime}}{\alpha}-\frac{\beta^{\prime}}{\beta}\right)}=i \frac{|\alpha|^{2} \sqrt{\bar{\alpha} \bar{\beta}}}{\alpha \beta}\left(\frac{\alpha^{\prime}}{\alpha}-\frac{\beta^{\prime}}{\beta}\right), \\
\left(\frac{\dot{\gamma}}{\sqrt{\alpha \beta}}\right) & =\frac{i|\alpha|^{2}}{\sqrt{\bar{\alpha} \bar{\beta}}}\left(\frac{\alpha^{\prime}}{\alpha}-\frac{\beta^{\prime}}{\beta}\right)
\end{aligned}
$$

at $p=\gamma(0)$ because (3.5) and $\tilde{\delta}_{d}(0)=0$. Hence we have

$$
\begin{aligned}
\left.\frac{d}{d t} \tilde{\delta}_{d}\right|_{t=0} & =\frac{1}{2}\left(\frac{\dot{\gamma}}{\sqrt{\alpha \beta}}(S(\alpha)-S(\beta))+\overline{\left(\frac{\dot{\gamma}}{\sqrt{\alpha \beta}}(S(\alpha)-S(\beta))\right)}\right)(p) \\
& =i|\alpha|^{2} \sqrt{\bar{\alpha} \bar{\beta}}\left(\frac{\alpha^{\prime}}{\alpha}-\frac{\beta^{\prime}}{\beta}\right)\left(\frac{S(\alpha)-S(\beta)}{\alpha \beta}+\overline{\left(\frac{S(\alpha)-S(\beta)}{\alpha \beta}\right)}\right)(p) \\
& =\frac{i|\alpha|^{4}}{\sqrt{\alpha \beta}}\left(\frac{\alpha^{\prime}}{\alpha}-\frac{\beta^{\prime}}{\beta}\right) \operatorname{Re}\left(\frac{S(\alpha)-S(\beta)}{\alpha \beta}\right)(p) .
\end{aligned}
$$

We note that $\left.(d / d t) \delta_{d}\right|_{t=0} \neq 0$ is equivalent to $\left.(d / d t) \tilde{\delta}_{d}\right|_{t=0} \neq 0$ under the condition $\delta_{d}(0)=0$. Thus by [20, Proposition 1.3] again, we have the conclusion.

Remark 3.3. Assuming that $u(z)$ is a solution of the differential equation

$$
u^{\prime \prime}(z)-\alpha(z) u(z)=0,
$$

satisfying $\operatorname{det} A=1$, where $A=\left(u(z), u^{\prime}(z)\right)$. then the matrix $A$ satisfies (3.1). This equation (3.11) is called the $S L$-form of the hypergeometric equation. The flat fronts $A A^{*}$ and $A e_{3} A^{*}$ in $H^{3}$ and $S_{1}^{3}$ constructed from the data $(\alpha, 1)$ can be regarded generalizations of the Schwarz map of the hypergeometric equation. They are called hyperbolic and de Sitter Schwarz map (31, see also [4,32]). Thus setting $\beta=1$, then all invariants given in Section 3 can be regarded as invariants of these Schwarz map.

3.2. Geometric invariants of cuspidal edges. For flat fronts $f: U \rightarrow H^{3}$ and $g: U \rightarrow S_{1}^{3}$ given by (3.2). Let $p \in U$ be a singular point, and let $f$ at $p$ be a cuspidal edge. Let $\gamma(t)$ be a parametrization of $\Sigma(f)$ or $\Sigma(g)$. Let $\kappa_{s}^{h}, \kappa_{t}^{h}$ and $\kappa_{c}^{h}$ (respectively, $\kappa_{s}^{d}, \kappa_{t}^{d}$ and $\kappa_{c}^{d}$ ) be the singular curvature, cuspidal torsion and the cuspidal curvature of $f$ on $\Sigma(f)$ (respectively, of $g$ on $\Sigma(g)$ ). Then we have the following theorem. 
Theorem 3.4. It holds that

$$
\begin{array}{ll}
\kappa_{s}^{h}(t)=-\left.\frac{\left|\lambda^{\prime}\right|^{2}}{4|\alpha|^{2}\left|\operatorname{Im}\left(\sqrt{\alpha \beta} \lambda_{\bar{z}}\right)\right|}\right|_{(u, v)=\gamma(t)} & =-\left.\frac{\left|\lambda^{\prime}\right|^{2}}{4|\alpha|^{4} C_{h}}\right|_{(u, v)=\gamma(t)} \\
\kappa_{t}^{h}(t)=\left.\frac{\operatorname{Re}\left(\sqrt{\alpha \beta} \lambda_{\bar{z}}\right)}{\operatorname{Im}\left(\sqrt{\alpha \beta} \lambda_{\bar{z}}\right)}\right|_{(u, v)=\gamma(t)} & =\left.\frac{C_{d}}{C_{h}}\right|_{(u, v)=\gamma(t)} \\
\kappa_{c}^{h}(t)=\left.\frac{4|\alpha|^{2} \operatorname{Im}\left(\sqrt{\alpha \beta} \lambda_{\bar{z}}\right)}{\left|\operatorname{Im}\left(\sqrt{\alpha \beta} \lambda_{\bar{z}}\right)\right|^{3 / 2}}\right|_{(u, v)=\gamma(t)} & =\left.\frac{4|\alpha| C_{h}}{\left|C_{h}\right|^{3 / 2}}\right|_{(u, v)=\gamma(t)}
\end{array}
$$

and

$$
\begin{aligned}
\kappa_{s}^{d}(t)=-\left.\frac{\left|\lambda^{\prime}\right|^{2}}{4|\alpha|^{2}\left|\operatorname{Re}\left(\sqrt{\alpha \beta} \lambda_{\bar{z}}\right)\right|}\right|_{(u, v)=\gamma(t)} & =-\left.\frac{\left|\lambda^{\prime}\right|^{2}}{4|\alpha|^{4} C_{d}}\right|_{(u, v)=\gamma(t)} \\
\kappa_{t}^{d}(t)=-\left.\frac{\operatorname{Im}\left(\sqrt{\alpha \beta} \lambda_{\bar{z}}\right)}{\operatorname{Re}\left(\sqrt{\alpha \beta} \lambda_{\bar{z}}\right)}\right|_{(u, v)=\gamma(t)} & =-\left.\frac{C_{h}}{C_{d}}\right|_{(u, v)=\gamma(t)} \\
\kappa_{c}^{d}(t)=-\left.\frac{4|\alpha|^{2} \operatorname{Re}\left(\sqrt{\alpha \beta} \lambda_{\bar{z}}\right)}{\left|\operatorname{Re}\left(\sqrt{\alpha \beta} \lambda_{\bar{z}}\right)\right|^{3 / 2}}\right|_{(u, v)=\gamma(t)} & =-\left.\frac{4 \mid \alpha C_{d}}{\left|C_{d}\right|^{3 / 2}}\right|_{(u, v)=\gamma(t)} .
\end{aligned}
$$

In particular, both $\kappa_{s}^{h}$ and $\kappa_{s}^{d}$ are strictly negative if $f$ and $g$ have only cuspidal edges.

We can observe dual relations between $\kappa_{t}^{h}$ and $\kappa_{t}^{d}$ and between $\kappa_{t}^{h}$ and $C_{d}$ (respectively, $\kappa_{t}^{d}$ and $C_{h}$ ). We will see this duality in Section 3.3. We remark that since the Gaussian curvature is bounded, $\kappa_{\nu}$ vanishes identically for $f$ and $g$.

Proof. We first calculate the invariant $\kappa_{s}$ for $f$ and $g$. By a direct calculation, we have

$$
\begin{aligned}
& \xi f=i A\left(\begin{array}{cc}
0 & -\lambda_{\bar{z}} \alpha+\lambda^{\prime} \bar{\beta} \\
-\lambda_{\bar{z}} \beta+\lambda^{\prime} \bar{\alpha} & 0
\end{array}\right) A^{*}, \\
& \xi g=i A\left(\begin{array}{cc}
0 & \lambda_{\bar{z}} \alpha+\lambda^{\prime} \bar{\beta} \\
-\left(\lambda_{\bar{z}} \beta+\lambda^{\prime} \bar{\alpha}\right) & 0
\end{array}\right) A^{*},
\end{aligned}
$$

and on the singular set,

$$
\begin{gathered}
\xi f=i \frac{-\lambda_{\bar{z}} \alpha+\lambda^{\prime} \bar{\beta}}{\alpha} A D A^{*}=2 \bar{\beta} \sqrt{\frac{\beta}{\alpha}} C_{h} A D A^{*}, \\
\xi g=i \frac{\lambda_{\bar{z}} \beta+\lambda^{\prime} \bar{\alpha}}{\beta} A D e_{3} A^{*}=2 i \sqrt{\bar{\alpha} \bar{\beta}} C_{d} A D e_{3} A^{*} .
\end{gathered}
$$

Thus on the singular set, we have

$$
\begin{aligned}
\nabla_{\xi} \xi f & \equiv-2 i \bar{\beta} \sqrt{\frac{\beta}{\alpha}} C_{h} A \tilde{D}_{h} A^{*} \bmod \langle f\rangle_{\mathcal{E}(U)}, \\
\nabla_{\xi} \xi g & \equiv-2 i \bar{\alpha} \sqrt{\frac{\alpha}{\beta}} C_{d} A \tilde{D}_{d} A^{*} \bmod \langle g\rangle_{\mathcal{E}(U)},
\end{aligned}
$$

where

$$
\tilde{D}_{h}=-\lambda_{\bar{z}} D^{2}-\lambda_{\bar{z}} D^{\prime}+\lambda^{\prime} D D^{*}, \quad \tilde{D}_{d}=-\lambda_{\bar{z}} D^{2} e_{3}-\lambda_{\bar{z}} D^{\prime} e_{3}+\lambda^{\prime} D e_{3} D^{*} .
$$


Here $\mathcal{E}(U)=\{h: U \rightarrow \boldsymbol{R}\}$ is the ring consists of function-germs on $U$, and $\left\langle k_{1}, \ldots, k_{r}\right\rangle_{\mathcal{E}(U)}=\left\{a_{1} k_{1}+\cdots+a_{r} k_{r} \mid a_{1}, \ldots, a_{r} \in \mathcal{E}(U)\right\}$. The formula $f_{1} \equiv f_{2}$ mod $A$ stands for $f_{1}-f_{2} \in A$. Hence, we have

$$
\xi f \times \nabla_{\xi} \xi f \equiv 4 \bar{\beta}^{2} \frac{\alpha}{\beta} C_{h}^{2} \lambda_{\bar{z}}\left(\alpha \beta^{\prime}-\alpha^{\prime} \beta\right) g \equiv-4 \bar{\beta}^{2} \frac{\alpha^{2}}{\beta \bar{\beta}} C_{h}^{2}\left|\lambda^{\prime}\right|^{2} g \quad \bmod \langle\xi f \times f\rangle_{\mathcal{E}(U)}
$$

and

$$
\xi g \times \nabla_{\xi} \xi g \equiv-4 \bar{\alpha} \bar{\beta} C_{d}^{2} \lambda_{\bar{z}}\left(\alpha \beta^{\prime}-\alpha^{\prime} \beta\right) f \equiv 4 \alpha \bar{\alpha} C_{d}^{2}\left|\lambda^{\prime}\right|^{2} f \quad \bmod \langle\xi g \times g\rangle_{\mathcal{E}(U)}
$$

on the singular set, where we see $\lambda_{\bar{z}}\left(\alpha \beta^{\prime}-\alpha^{\prime} \beta\right)=-\lambda_{\bar{z}}(\alpha / \bar{\beta})\left(\alpha \alpha^{\prime}-\beta \beta^{\prime}\right)$ on the singular set. On the other hand, the signed area density function for $f$ and $g$ are $\Lambda_{h}=\Omega\left(\xi f, \eta_{h} f, g\right)$ and $\Lambda_{d}=\Omega\left(\xi g, \eta_{d} g, f\right)$, respectively. Then we see that $\eta_{h} \Lambda_{h}=-\left|\lambda_{\eta_{h}}\right|^{2}$, and $\eta_{d} \Lambda_{d}=\left|\lambda_{\eta_{d}}\right|^{2}$. Thus we have (3.12) and (3.15).

Now we proceed to calculate the cuspidal torsion and the cuspidal curvature. To show this, we prove several formulas of differentials of $f$ and $g$ needed later.

Lemma 3.5. On the singular set, it holds that

$$
f^{\prime} \times f_{\bar{z}}=f^{\prime} \times f_{\bar{z}}^{\prime}=f_{\bar{z}} \times f_{\bar{z}}^{\prime}=g^{\prime} \times g_{\bar{z}}=g^{\prime} \times g_{\bar{z}}^{\prime}=g_{\bar{z}} \times g_{\bar{z}}^{\prime}=0
$$

and

$$
\begin{aligned}
f^{\prime} \times f^{\prime \prime} & =\frac{i}{2}\left(\alpha \beta^{\prime}-\alpha^{\prime} \beta\right) g, \quad f^{\prime} \times f_{\overline{z z}}=\frac{i}{2} \lambda_{\bar{z}} g, \\
f_{\bar{z}} \times f^{\prime \prime} & =-\frac{i}{2} \lambda^{\prime} g, \quad f_{\bar{z}} \times f_{\overline{z z}}=\frac{i}{2} \overline{\left(\alpha^{\prime} \beta-\alpha \beta^{\prime}\right)} g, \\
g^{\prime} \times g^{\prime \prime} & =\frac{i}{2}\left(\alpha \beta^{\prime}-\alpha^{\prime} \beta\right) f, \quad g^{\prime} \times g_{\overline{z z}}=-\frac{i}{2} \lambda_{\bar{z}} f, \\
g_{\bar{z}} \times g^{\prime \prime} & =\frac{i}{2} \lambda^{\prime} f, \quad g_{\bar{z}} \times g_{\overline{z z}}=\frac{i}{2} \overline{\left(\alpha^{\prime} \beta-\alpha \beta^{\prime}\right)} f .
\end{aligned}
$$

In particular, all vectors in (3.20) are parallel to $g$, and that in (3.21) are parallel to $f$.

Proof. By a direct calculation, we have

$$
f^{\prime \prime}=A\left(\begin{array}{cc}
\alpha \beta & \alpha^{\prime} \\
\beta^{\prime} & \alpha \beta
\end{array}\right) A^{*}, f_{\bar{z}}^{\prime}=A\left(\begin{array}{cc}
|\alpha|^{2} & 0 \\
0 & |\beta|^{2}
\end{array}\right) A^{*}, f_{\overline{z z}}=A\left(\begin{array}{cc}
\bar{\alpha} \bar{\beta} & \overline{\beta^{\prime}} \\
\overline{\alpha^{\prime}} & \bar{\alpha} \bar{\beta}
\end{array}\right) A^{*}
$$

and

$$
g^{\prime \prime}=A\left(\begin{array}{cc}
\alpha \beta & -\alpha^{\prime} \\
\beta^{\prime} & -\alpha \beta
\end{array}\right) A^{*}, g_{\bar{z}}^{\prime}=A\left(\begin{array}{cc}
-|\alpha|^{2} & 0 \\
0 & |\beta|^{2}
\end{array}\right) A^{*}, g_{\overline{z z}}=A\left(\begin{array}{cc}
\bar{\alpha} \bar{\beta} & \overline{\beta^{\prime}} \\
-\overline{\alpha^{\prime}} & -\bar{\alpha} \bar{\beta}
\end{array}\right) A^{*} .
$$

This shows the assertion.

Lemma 3.6. On the singular set, it holds that

$$
\begin{gathered}
\xi f \times \nabla_{\eta_{h}} \eta_{h} f=\frac{2}{|\alpha|^{4}}\left(\operatorname{Im}\left(\sqrt{\alpha \beta} \lambda_{\bar{z}}\right)\right)^{2} g, \\
\xi f \times \nabla_{\eta_{d}} \eta_{d} g=\frac{-2}{|\alpha|^{4}}\left(\operatorname{Re}\left(\sqrt{\alpha \beta} \lambda_{\bar{z}}\right)\right)^{2} f .
\end{gathered}
$$

Proof. By definition, we have

$$
\nabla_{\eta_{h}} \eta_{h} f=\eta_{h} \eta_{h} f+\left\langle\eta_{h} \eta_{h} f, f\right\rangle f, \quad \nabla_{\eta_{d}} \eta_{d} g=\eta_{d} \eta_{d} g-\left\langle\eta_{d} \eta_{d} g, g\right\rangle g .
$$


Since $\left\langle\eta_{h} f, f\right\rangle=\left\langle\eta_{d} g, g\right\rangle=0$, it holds that $\left\langle\eta_{h} \eta_{h} f, f\right\rangle+\left\langle\eta_{h} f, \eta_{h} f\right\rangle=0$ and $\left\langle\eta_{d} \eta_{d} g, g\right\rangle+\left\langle\eta_{d} g, \eta_{d} g\right\rangle=0$. Moreover, on the singular set, $\eta_{h} f=\eta_{d} g=0$, and hence $\left\langle\eta_{h} \eta_{h} f, f\right\rangle=\left\langle\eta_{d} \eta_{d} g, g\right\rangle=0$ on $\Sigma(f)=\Sigma(g)$. In particular, it holds that $\nabla_{\eta_{h}} \eta_{h} f=\eta_{h} \eta_{h} f$ and $\nabla_{\eta_{d}} \eta_{d} g=\eta_{d} \eta_{d} g$, and we have $\xi f \times \nabla_{\eta_{h}} \eta_{h} f=\xi f \times \eta_{h} \eta_{h} f$ and $\xi g \times \nabla_{\eta_{d}} \eta_{d} g=\xi g \times \eta_{d} \eta_{d} g$ on the singular set.

On the singular set, it follows that

$$
\begin{aligned}
& \eta_{h} \eta_{h} f=\frac{-f^{\prime \prime}}{\alpha \beta}+\frac{2 f_{\bar{z}}^{\prime}}{|\alpha|^{2}}-\frac{f_{\overline{z z}}}{\bar{\alpha} \bar{\beta}}+\frac{1}{2 \alpha \beta}\left(\frac{\alpha^{\prime}}{\alpha}+\frac{\beta^{\prime}}{\beta}\right) f^{\prime}+\frac{1}{2 \bar{\alpha} \bar{\beta}} \overline{\left(\frac{\alpha^{\prime}}{\alpha}+\frac{\beta^{\prime}}{\beta}\right)} f_{\bar{z}} \\
& \eta_{d} \eta_{d} g=\frac{g^{\prime \prime}}{\alpha \beta}+\frac{2 g_{\bar{z}}^{\prime}}{|\alpha|^{2}}+\frac{g_{\overline{z z}}}{\bar{\alpha} \bar{\beta}}-\frac{1}{2 \alpha \beta}\left(\frac{\alpha^{\prime}}{\alpha}+\frac{\beta^{\prime}}{\beta}\right) g^{\prime}-\frac{1}{2 \bar{\alpha} \bar{\beta}} \overline{\left(\frac{\alpha^{\prime}}{\alpha}+\frac{\beta^{\prime}}{\beta}\right)} g_{\bar{z}} .
\end{aligned}
$$

By (3.19), (3.20), (3.21), (3.24), (3.25) and the relation $|\alpha|^{2}\left(\alpha^{\prime} \beta-\alpha \beta^{\prime}\right)=\alpha \beta \lambda^{\prime}$ which holds on $\Sigma(f)=\Sigma(g)$, we have the assertion.

By Lemma 3.6 for any $X \in T_{f(p)} H^{3}$ and $Y \in T_{g(p)} S_{1}^{3}$, we have

$$
\begin{aligned}
& \Omega\left(\xi f, \nabla_{\eta_{h}} \eta_{h} f, X\right)=\frac{2}{|\alpha|^{4}}\left(\operatorname{Im}\left(\sqrt{\alpha \beta} \lambda_{\bar{z}}\right)\right)^{2}\langle g, X\rangle, \\
& \Omega\left(\xi g, \nabla_{\eta_{d}} \eta_{d} g, Y\right)=\frac{-2}{|\alpha|^{4}}\left(\operatorname{Re}\left(\sqrt{\alpha \beta} \lambda_{\bar{z}}\right)\right)^{2}\langle f, Y\rangle .
\end{aligned}
$$

Lemma 3.7. On the singular set, $\left\langle\nabla_{\eta_{h}} \eta_{h} f, \xi f\right\rangle=\left\langle\nabla_{\eta_{d}} \eta_{d} g, \xi g\right\rangle=0$ holds.

Proof. By the above arguments, it follows that $\left\langle\nabla_{\eta_{h}} \eta_{h} f, \xi f\right\rangle=\left\langle\eta_{h} \eta_{h} f, \xi f\right\rangle$ and $\left\langle\nabla_{\eta_{d}} \eta_{d} g, \xi g\right\rangle=\left\langle\eta_{d} \eta_{d} g, \xi g\right\rangle$ on $\Sigma(f)=\Sigma(g)$. By (3.22) and (3.23),

$$
\begin{aligned}
\left\langle f^{\prime}, f^{\prime}\right\rangle & =\alpha \beta, \quad\left\langle f^{\prime}, f_{\bar{z}}\right\rangle=|\alpha|^{2}, \quad\left\langle f_{\bar{z}}, f_{\bar{z}}\right\rangle=\bar{\alpha} \bar{\beta}, \\
\left\langle f^{\prime}, f^{\prime \prime}\right\rangle & =\frac{1}{2}\left(\alpha^{\prime} \beta+\alpha \beta^{\prime}\right), \quad\left\langle f^{\prime}, f_{\bar{z}}^{\prime}\right\rangle=0, \quad\left\langle f^{\prime}, f_{\overline{z z}}\right\rangle=\frac{1}{2}\left(\alpha \overline{\alpha^{\prime}}+\beta \overline{\beta^{\prime}}\right), \\
\left\langle f_{\bar{z}}, f^{\prime \prime}\right\rangle & =\frac{1}{2}\left(\alpha^{\prime} \bar{\alpha}+\beta^{\prime} \bar{\beta}\right), \quad\left\langle f_{\bar{z}}, f_{\bar{z}}^{\prime}\right\rangle=0, \quad\left\langle f_{\bar{z}}, f_{\overline{z z}}\right\rangle=\frac{1}{2} \overline{\left(\alpha^{\prime} \beta+\alpha \beta^{\prime}\right)}, \\
\left\langle g^{\prime}, g^{\prime}\right\rangle & =-\alpha \beta, \quad\left\langle g^{\prime}, g_{\bar{z}}\right\rangle=|\alpha|^{2}, \quad\left\langle g_{\bar{z}}, g_{\bar{z}}\right\rangle=-\bar{\alpha} \bar{\beta} \\
\left\langle g^{\prime}, g^{\prime \prime}\right\rangle & =\frac{-1}{2}\left(\alpha^{\prime} \beta+\alpha \beta^{\prime}\right), \quad\left\langle g^{\prime}, g_{\bar{z}}^{\prime}\right\rangle=0, \quad\left\langle g^{\prime}, g_{\bar{z} \bar{z}}\right\rangle=\frac{1}{2}\left(\alpha \overline{\alpha^{\prime}}+\beta \overline{\beta^{\prime}}\right), \\
\left\langle g_{\bar{z}}, g^{\prime \prime}\right\rangle & =\frac{1}{2}\left(\alpha^{\prime} \bar{\alpha}+\beta^{\prime} \bar{\beta}\right), \quad\left\langle g_{\bar{z}}, g_{\bar{z}}^{\prime}\right\rangle=0, \quad\left\langle g_{\bar{z}}, g \overline{z \bar{z}}\right\rangle=\frac{-1}{2} \overline{\left(\alpha^{\prime} \beta+\alpha \beta^{\prime}\right)}
\end{aligned}
$$

hold on the singular set. Moreover, we have $\alpha^{\prime} \bar{\alpha}+\beta^{\prime} \bar{\beta}=|\alpha|^{2}\left(\alpha^{\prime} / \alpha+\beta^{\prime} / \beta\right)$, $\alpha^{\prime} \beta+\alpha \beta^{\prime}=\alpha \beta\left(\alpha^{\prime} / \alpha+\beta^{\prime} / \beta\right)$ on the singular set. Thus we have $\left\langle\eta_{h} \eta_{h} f, \xi f\right\rangle=$ $\left\langle\eta_{d} \eta_{d} g, \xi g\right\rangle=0$ on the singular set.

We turn to calculate $\kappa_{t}^{h}$ and $\kappa_{t}^{d}$. By Lemma 3.7, the second terms of $\kappa_{t}^{h}$ and $\kappa_{t}^{d}$ in (2.1) vanish. Thus for the calculations of them, only the first terms of $\kappa_{t}^{h}$ and $\kappa_{t}^{d}$ in (2.1) are needed. Since $\left\langle f^{\prime \prime}, g\right\rangle=\left\langle f_{\bar{z}}^{\prime}, g\right\rangle=\left\langle f_{\overline{z z}}, g\right\rangle=0$ and $\left\langle g^{\prime \prime}, f\right\rangle=\left\langle g_{\bar{z}}^{\prime}, f\right\rangle=$ 
$\left\langle g_{\overline{z z}}, f\right\rangle=0$ hold on $\Sigma(f)=\Sigma(g)$, and by (3.24) and (3.25), it holds that

$$
\begin{aligned}
\nabla_{\xi} \nabla_{\eta_{h}} \eta_{h} f & \equiv-i \lambda_{\bar{z}}\left(\frac{-1}{\alpha \beta} f^{\prime \prime \prime}+\frac{2}{|\alpha|^{2}} f_{\bar{z}}^{\prime \prime}-\frac{1}{\bar{\alpha} \bar{\beta}} f_{\overline{z z}}^{\prime}\right) \\
& +i \lambda^{\prime}\left(\frac{-1}{\alpha \beta} f_{\bar{z}}^{\prime \prime}+\frac{2}{|\alpha|^{2}} f_{\overline{z z}}^{\prime}-\frac{1}{\bar{\alpha} \bar{\beta}} f_{\overline{z z z}}\right) \bmod \mathcal{F}, \\
\nabla_{\xi} \nabla_{\eta_{d}} \eta_{d} g & \equiv-i \lambda_{\bar{z}}\left(\frac{1}{\alpha \beta} g^{\prime \prime \prime}+\frac{2}{|\alpha|^{2}} g_{\bar{z}}^{\prime \prime}+\frac{1}{\bar{\alpha} \bar{\beta}} g_{\overline{z z}}^{\prime}\right) \\
& +i \lambda^{\prime}\left(\frac{1}{\alpha \beta} g_{\bar{z}}^{\prime \prime}+\frac{2}{|\alpha|^{2}} g_{\overline{z z}}^{\prime}+\frac{1}{\bar{\alpha} \bar{\beta}} g_{\overline{z z z}}\right) \bmod \mathcal{G},
\end{aligned}
$$

where $\mathcal{F}=\left\langle f, f^{\prime} f_{\bar{z}}, f^{\prime \prime}, f_{\bar{z}}^{\prime}, f_{\overline{z z}}\right\rangle_{\mathcal{E}(U)}, \mathcal{G}=\left\langle g, g^{\prime} g_{\bar{z}}, g^{\prime \prime}, g_{\bar{z}}^{\prime}, g_{\overline{z z}}\right\rangle_{\mathcal{E}(U)}$. We show the following lemma:

Lemma 3.8. On the singular set, it holds that

$$
\begin{aligned}
& \left\langle f^{\prime \prime \prime}, g\right\rangle=\frac{-\alpha \beta}{2|\alpha|^{2}} \lambda^{\prime}, \quad\left\langle f_{\bar{z}}^{\prime \prime}, g\right\rangle=\frac{\lambda^{\prime}}{2}, \quad\left\langle f_{\overline{z z}}^{\prime}, g\right\rangle=\frac{\lambda_{\bar{z}}}{2}, \quad\left\langle f_{\overline{z z z}}, g\right\rangle=\frac{-\bar{\alpha} \bar{\beta}}{2|\alpha|^{2}} \lambda_{\bar{z}}, \\
& \left\langle g^{\prime \prime \prime}, f\right\rangle=\frac{\alpha \beta}{2|\alpha|^{2}} \lambda^{\prime}, \quad\left\langle g_{\bar{z}}^{\prime \prime}, f\right\rangle=\frac{\lambda^{\prime}}{2}, \quad\left\langle g_{\overline{z z}}^{\prime}, f\right\rangle=\frac{\lambda_{\bar{z}}}{2}, \quad\left\langle g_{\overline{z z z}}, f\right\rangle=\frac{\bar{\alpha} \bar{\beta}}{2|\alpha|^{2}} \lambda_{\bar{z}} .
\end{aligned}
$$

Proof. By a direct calculation, we have

$$
\begin{aligned}
& f^{\prime \prime \prime}=A\left(\begin{array}{cc}
\alpha^{\prime} \beta+2 \alpha \beta^{\prime} & \alpha^{\prime \prime}+\alpha^{2} \beta \\
\beta^{\prime \prime}+\alpha \beta^{2} & 2 \alpha^{\prime} \beta+\alpha \beta^{\prime}
\end{array}\right) A^{*}, f_{\bar{z}}^{\prime \prime}=A\left(\begin{array}{cc}
\alpha^{\prime} \bar{\alpha} & \alpha|\beta|^{2} \\
\beta|\alpha|^{2} & \beta^{\prime} \bar{\beta}
\end{array}\right) A^{*}, \\
& f_{\overline{z z}}^{\prime}=A\left(\begin{array}{cc}
\alpha \overline{\alpha^{\prime}} & \bar{\beta}|\alpha|^{2} \\
\bar{\alpha}|\beta|^{2} & \left.\beta\right|^{\prime}
\end{array}\right) A^{*}, f_{\overline{z z z}}=A\left(\begin{array}{cc}
\overline{\alpha^{\prime} \beta}+2 \bar{\alpha} \overline{\beta^{\prime}} & \overline{\beta^{\prime \prime}}+\bar{\alpha}^{2} \\
\overline{\alpha^{\prime \prime}}+\bar{\alpha}^{2} \bar{\beta} & 2 \overline{\alpha^{\prime} \beta}+\bar{\alpha} \bar{\beta}^{\prime}
\end{array}\right) A^{*},
\end{aligned}
$$

and

$$
\begin{aligned}
& g^{\prime \prime \prime}=A\left(\begin{array}{cc}
\alpha^{\prime} \beta+2 \alpha \beta^{\prime} & -\left(\alpha^{\prime \prime}+\alpha^{2} \beta\right) \\
\beta^{\prime \prime}+\alpha \beta^{2} & -\left(2 \alpha^{\prime} \beta+\alpha \beta^{\prime}\right)
\end{array}\right) A^{*}, g_{\bar{z}}^{\prime \prime}=A\left(\begin{array}{cc}
-\alpha^{\prime} \bar{\alpha} & \alpha|\beta|^{2} \\
-\beta|\alpha|^{2} & \beta^{\prime} \bar{\beta}
\end{array}\right) A^{*}, \\
& g_{\overline{z z}}^{\prime}=A\left(\begin{array}{cc}
-\alpha \overline{\alpha^{\prime}} & -\bar{\beta}|\alpha|^{2} \\
\bar{\alpha}|\beta|^{2} & \beta \overline{\beta^{\prime}}
\end{array}\right) A^{*}, g_{\overline{z z z}}=A\left(\begin{array}{cc}
\overline{\alpha^{\prime} \beta}+2 \bar{\alpha} \overline{\beta^{\prime}} & \overline{\beta^{\prime \prime}}+\bar{\alpha} \overline{\beta^{2}} \\
\left.-\overline{\alpha^{\prime \prime}}+\overline{\alpha^{2}} \bar{\beta}\right) & -\left(2 \overline{\alpha^{\prime} \beta}+\bar{\alpha} \overline{\beta^{\prime}}\right)
\end{array}\right) A^{*} .
\end{aligned}
$$

This shows the assertion.

Let us continue the calculations for $\kappa_{t}^{h}$ and $\kappa_{t}^{d}$. By (3.26) and (3.27),

$$
\begin{aligned}
\Omega\left(\xi f, \nabla_{\eta_{h}} \eta_{h} f, \nabla_{\xi} \nabla_{\eta_{h}} \eta_{h} f\right) & =\frac{i\left(\operatorname{Im}\left(\sqrt{\alpha \beta} \lambda_{\bar{z}}\right)\right)^{2}}{|\alpha|^{8}}\left(\alpha \beta\left(\lambda_{\bar{z}}\right)^{2}-\bar{\alpha} \bar{\beta}\left(\lambda^{\prime}\right)^{2}\right) \\
& =\frac{4 \operatorname{Re}\left(\sqrt{\alpha \beta} \lambda_{\bar{z}}\right)\left(\operatorname{Im}\left(\sqrt{\alpha \beta} \lambda_{\bar{z}}\right)\right)^{3}}{|\alpha|^{8}}, \\
\Omega\left(\xi g, \nabla_{\eta_{d}} \eta_{d} g, \nabla_{\xi} \nabla_{\eta_{d}} \eta_{d} g\right) & =\frac{i\left(\operatorname{Re}\left(\sqrt{\alpha \beta} \lambda_{\bar{z}}\right)\right)^{2}}{|\alpha|^{8}}\left(\alpha \beta\left(\lambda_{\bar{z}}\right)^{2}-\bar{\alpha} \bar{\beta}\left(\lambda^{\prime}\right)^{2}\right) \\
& =\frac{4 \operatorname{Im}\left(\sqrt{\alpha \beta} \lambda_{\bar{z}}\right)\left(\operatorname{Re}\left(\sqrt{\alpha \beta} \lambda_{\bar{z}}\right)\right)^{3}}{|\alpha|^{8}}
\end{aligned}
$$


hold on the singular set. Therefore by (2.1), the formulas (3.13) and (3.16), are proven. Finally, we consider the cuspidal curvatures $\kappa_{c}^{h}$ and $\kappa_{c}^{d}$. By (3.18),

$$
|\xi f|=2|\alpha|^{2}\left|C_{h}\right|=2\left|\operatorname{Im}\left(\sqrt{\alpha \beta} \lambda_{\bar{z}}\right)\right|, \quad|\xi g|=2|\alpha|^{2}\left|C_{d}\right|=2\left|\operatorname{Re}\left(\sqrt{\alpha \beta} \lambda_{\bar{z}}\right)\right|
$$

hold on the singular set. Moreover, we have

$$
\begin{aligned}
\nabla_{\eta_{h}} \nabla_{\eta_{h}} \eta_{h} f & \equiv \frac{i}{\sqrt{\alpha \beta}}\left(\frac{-1}{\alpha \beta} f^{\prime \prime \prime}+\frac{2}{|\alpha|^{2}} f_{\bar{z}}^{\prime \prime}-\frac{1}{\bar{\alpha} \bar{\beta}} f_{\overline{z z}}^{\prime}\right) \\
& -\frac{i}{\sqrt{\bar{\alpha} \bar{\beta}}}\left(\frac{-1}{\alpha \beta} f_{\bar{z}}^{\prime \prime}+\frac{2}{|\alpha|^{2}} f_{\overline{z z}}^{\prime}-\frac{1}{\bar{\alpha} \bar{\beta}} f_{\overline{z z z}}\right) \bmod \mathcal{F}, \\
\nabla_{\eta_{d}} \nabla_{\eta_{d}} \eta_{d} g & \equiv \frac{1}{\sqrt{\alpha \beta}}\left(\frac{1}{\alpha \beta} g^{\prime \prime \prime}+\frac{2}{|\alpha|^{2}} g_{\bar{z}}^{\prime \prime}+\frac{1}{\bar{\alpha} \bar{\beta}} g_{\overline{z z}}^{\prime}\right) \\
& +\frac{1}{\sqrt{\bar{\alpha} \bar{\beta}}}\left(\frac{1}{\alpha \beta} g_{\bar{z}}^{\prime \prime}+\frac{2}{|\alpha|^{2}} g_{\overline{z z}}^{\prime}+\frac{1}{\bar{\alpha} \bar{\beta}} g_{\overline{z z z}}\right) \bmod \mathcal{G}
\end{aligned}
$$

on the singular set. Thus we have

$$
\begin{aligned}
& \left\langle g, \nabla_{\eta_{h}} \nabla_{\eta_{h}} \eta_{h} f\right\rangle=\frac{2 i}{|\alpha|^{2}}\left(\frac{\lambda^{\prime}}{\sqrt{\alpha \beta}}-\frac{\lambda_{\bar{z}}}{\sqrt{\bar{\alpha} \bar{\beta}}}\right)=\frac{4 \operatorname{Im}\left(\sqrt{\alpha \beta} \lambda_{\bar{z}}\right)}{|\alpha|^{4}} \\
& \left\langle f, \nabla_{\eta_{d}} \nabla_{\eta_{d}} \eta_{d} g\right\rangle=\frac{2}{|\alpha|^{2}}\left(\frac{\lambda^{\prime}}{\sqrt{\alpha \beta}}+\frac{\lambda_{\bar{z}}}{\sqrt{\bar{\alpha} \bar{\beta}}}\right)=\frac{4 \operatorname{Re}\left(\sqrt{\alpha \beta} \lambda_{\bar{z}}\right)}{|\alpha|^{4}}
\end{aligned}
$$

on the singular set by (3.27). Hence we obtain (3.14) and (3.17).

We note that $\kappa_{s}^{h}<0$ follows from the general theory (29, Theorem 3.1]) since the extrinsic Gaussian curvature of a flat front $f$ is $1>0$. However, $\kappa_{s}^{d}<0$ does not follow by the general theory since the extrinsic Gaussian curvature of a flat front $g$ is $-1<0$.

3.3. Relationships between curvatures and singularities of the dual surfaces. For a non-degenerate singular point $p$ of $f$ (resp. $g$ ), it is known that $f$ at $p$ (resp. $g$ at $p$ ) is not a cuspidal edge if and only if $C_{h}(p)=0\left(\right.$ resp. $\left.C_{d}(p)=0\right)$, where $C_{h}$ (resp. $C_{d}$ ) is as in (3.7) (resp. (3.8)) (cf. [20]). By Theorem [3.4 it follows that a flat front $f$ (resp. $g$ ) in $H^{3}$ (resp. $S_{1}^{3}$ ) is not a cuspidal edge at a non-degenerate singular point $p$ if and only if $\kappa_{t}^{d}\left(\right.$ resp. $\left.\kappa_{t}^{h}\right)$ vanishes at $p$. Therefore we consider relation between the type of singularity which is not a cuspidal edge of $f$ or $g$ and behavior of $\kappa_{t}^{d}$ or $\kappa_{t}^{h}$.

Let $U \subset C$ be a simply-connected domain and $\mathcal{O}(U)$ the set of holomorphic functions on $U$. Then, for $h \in \mathcal{O}(U)$, we can construct flat fronts

$$
f=f_{h}: U \rightarrow H^{3}, \quad g=g_{h}: U \rightarrow S_{1}^{3}
$$

which are represented by a pair of holomorphic functions $(\alpha, \beta)=\left(e^{h}, 1\right)$. Converse is also true, namely, for a flat front $f: U \rightarrow H^{3}$ without umbilical point, then we can choose a suitable complex coordinate $z$ such that $\alpha d z=e^{h} d z$ and $\beta d z=d z$. (see [20, p 323]).

Let $f: U \rightarrow H^{3}$ and $g: U \rightarrow S_{1}^{3}$ be flat fronts defined by (3.2) by the data $(\alpha, \beta)=\left(e^{h}, 1\right)$. Then the sets of singular points of $f$ and $g$ are

$$
\Sigma(f)=\Sigma(g)=\{z \in U \mid h(z)+\overline{h(z)}=0\} .
$$

Moreover, $\lambda=h+\bar{h}$ is an identifier of singularity. By Proposition 3.2, it holds that: 
- $f$ at $p(=\gamma(0))$ is a cuspidal edge if and only if $\operatorname{Im}\left(e^{-\frac{h}{2}} h^{\prime}\right) \neq 0$ at $p$,

- $f$ at $p$ is a swallowtail if and only if $\operatorname{Im}\left(e^{-\frac{h}{2}} h^{\prime}\right)=0$ and $\operatorname{Re}\left(e^{-h}\left(h^{\prime \prime}-\right.\right.$ $\left.\left.\frac{1}{2}\left(h^{\prime}\right)^{2}\right)\right) \neq 0$ at $p$.

- $g$ is a cuspidal edge at $p$ if and only if $\operatorname{Re}\left(e^{-\frac{h}{2}} h^{\prime}\right) \neq 0$ at $p$,

- $g$ is a swallowtail at $p$ if and only if $\operatorname{Re}\left(e^{-\frac{h}{2}} h^{\prime}\right)=0$ and $\operatorname{Re}\left(e^{-h}\left(h^{\prime \prime}-\right.\right.$ $\left.\left.\frac{1}{2}\left(h^{\prime}\right)^{2}\right)\right) \neq 0$ at $p$.

We have the following theorem.

Theorem 3.9. Let $f: U \rightarrow H^{3}$ and $g: U \rightarrow S_{1}^{3}$ be flat fronts and $p$ be a nondegenerate singular point of both $f$ and $g$. Let $\gamma(t)$ be a singular curve through $p=\gamma(0)$. Assume that $f$ at $p$ is a cuspidal edge and $\kappa_{t}^{h}(p)=0$ (resp. $g$ at $p$ is a cuspidal edge and $\left.\kappa_{t}^{d}(p)=0\right)$. Then $g($ resp. $f)$ is a swallowtail at $p$ if and only if $\left.\frac{d}{d t} \kappa_{t}^{h}\right|_{t=0} \neq 0\left(\right.$ resp. $\left.\left.\frac{d}{d t} \kappa_{t}^{d}\right|_{t=0} \neq 0\right)$.

Proof. Since this is a local situation, we may assume that both $f$ and $g$ are constructed by the data $(\alpha, \beta)=\left(e^{h}, 1\right)$, for $h \in \mathcal{O}(U)$. Let $\gamma(t)$ be a parametrization of $\Sigma(f)=\Sigma(g)$. By Theorem 3.4, $\kappa_{c}^{h}, \kappa_{c}^{d}, \kappa_{t}^{h}$ and $\kappa_{t}^{d}$ are given by

$$
\begin{aligned}
\kappa_{c}^{h}(t) & =\frac{4 \operatorname{Im}\left(e^{-\frac{h}{2}} h^{\prime}\right)}{\left|\operatorname{Im}\left(e^{-\frac{h}{2}} h^{\prime}\right)\right|^{3 / 2}}(\gamma(t)), & \kappa_{c}^{d}(t) & =-\frac{4 \operatorname{Re}\left(e^{-\frac{h}{2}} h^{\prime}\right)}{\left|\operatorname{Re}\left(e^{-\frac{h}{2}} h^{\prime}\right)\right|^{3 / 2}}(\gamma(t)), \\
\kappa_{t}^{h}(t) & =\frac{\operatorname{Re}\left(e^{-\frac{h}{2}} h^{\prime}\right)}{\operatorname{Im}\left(e^{-\frac{h}{2}} h^{\prime}\right)}(\gamma(t)), & \kappa_{t}^{d}(t) & =\frac{\operatorname{Im}\left(e^{-\frac{h}{2}} h^{\prime}\right)}{\operatorname{Re}\left(e^{-\frac{h}{2}} h^{\prime}\right)}(\gamma(t)) .
\end{aligned}
$$

First, we assume that $\kappa_{t}^{d}=0$ and $\kappa_{c}^{d} \neq 0$ at $p$. This is equivalent to the condition that $\operatorname{Im}\left(e^{-\frac{h}{2}} h^{\prime}\right)=0$ and $\operatorname{Re}\left(e^{-\frac{h}{2}} h^{\prime}\right) \neq 0$ at $p$. Thus $(d / d t) \kappa_{t}^{d}(0) \neq 0$ if and only if $(d / d t) \operatorname{Im}\left(e^{-\frac{h}{2}} h^{\prime}\right)(0) \neq 0$. By a direct calculation, we see that

$$
\begin{aligned}
& \frac{d}{d t}\left(\operatorname{Im}\left(e^{-\frac{h}{2}} h^{\prime}\right)(\gamma(t))\right) \\
= & \frac{1}{2 i}\left(e^{-\frac{h}{2}}\left(h^{\prime \prime}-\frac{1}{2}\left(h^{\prime}\right)^{2}\right) \dot{\gamma}+e^{-\frac{\bar{h}}{2}}\left(\overline{h^{\prime \prime}}-\frac{1}{2}\left(\overline{h^{\prime}}\right)^{2}\right) \dot{\gamma}\right)(t) \\
= & \frac{1}{2}\left(-e^{-\frac{h}{2}}\left(h^{\prime \prime}-\frac{1}{2}\left(h^{\prime}\right)^{2}\right) \overline{h^{\prime}}+e^{-\frac{\bar{h}}{2}}\left(\overline{h^{\prime \prime}}-\frac{1}{2}\left(\overline{h^{\prime}}\right)^{2}\right) h^{\prime}\right)(t) .
\end{aligned}
$$

Since we assume that $h+\bar{h}=0$ and $e^{-\frac{h}{2}} h^{\prime}-e^{-\frac{\bar{h}}{2}} \overline{h^{\prime}}=0$ at $p$, we have $\overline{h^{\prime}}=e^{-h} h^{\prime}$ and $h^{\prime}=e^{-\bar{h}} \overline{h^{\prime}}$. Hence it holds that

$$
\begin{aligned}
\left.\frac{d}{d t}\left(\operatorname{Im}\left(e^{-\frac{h}{2}} h^{\prime}\right)(\gamma(t))\right)\right|_{t=0}= & \frac{1}{2}\left(-e^{-h}\left(h^{\prime \prime}-\frac{1}{2}\left(h^{\prime}\right)^{2}\right) e^{-\frac{h}{2}} h^{\prime}\right. \\
& \left.+e^{-\bar{h}}\left(\overline{h^{\prime \prime}}-\frac{1}{2}\left(\overline{h^{\prime}}\right)^{2}\right) e^{-\frac{\bar{h}}{2}} \overline{h^{\prime}}\right)(p) \\
= & -\left(e^{-\frac{h}{2}} h^{\prime}\right)(p) \operatorname{Re}\left(\left(e^{-h}\left(h^{\prime \prime}-\frac{1}{2}\left(h^{\prime}\right)^{2}\right)\right)(p)\right),
\end{aligned}
$$

where we used the relation $e^{-\frac{h}{2}} h^{\prime}-e^{-\frac{h}{2}} \overline{h^{\prime}}=0$ at $p$ again to have the second equality. Thus we have the assertion for $f$.

The case for $g$ can be proven by the same computation using (3.30). 
In [22, Proposition 3.8], an equivalent statement of Theorem 3.9] is obtained from a different viewpoint. In fact, $\kappa_{t}^{h}$ coincides with $\Delta$ in [22, p. 1910] when $\varepsilon=0$ (a linear Weingarten front is a flat front if $\varepsilon=0$ ) in their notation. Thus Theorem 3.9 clarifies the geomteric meaning of $\Delta$ in $\left[22\right.$ for a flat front in $H^{3}$. In [22, it is shown that the co-orientabilities and orientabilities of the original front and its dual have a connection. Furthermore, zig-zag numbers of them are studied.

\section{Lines OF CURVATURE AND CONE-LIKE SINGULAR POINTS}

We consider the condition that the singular curve $\gamma$ of $f$ and $g$ are a line of curvature. Let $k: U \rightarrow N$ be a frontal into a Riemannian or semi-Riemannian 3-manifold $N$. Let $I$ be an interval. A curve $\gamma: I \rightarrow U$ is a line of curvature if $\mathrm{I}\left(k \circ \gamma^{\prime}\right)$ is parallel to $\mathbb{I}\left(k \circ \gamma^{\prime}\right)$, where $\mathrm{I}$ and $\mathrm{II}$ are the first and the second fundamental matrices, and they are regarded as linear maps $T_{p} \nu^{\perp} \rightarrow T_{p} \nu^{\perp} \subset T_{p} N$ and $k \circ \gamma^{\prime}(t) \in T_{p} \nu^{\perp}(p=\gamma(t))$.

Proposition 4.1. Let $f: U \rightarrow H^{3}$ and $g: U \rightarrow S_{1}^{3}$ be flat fronts constructed by (3.2). Let $p \in \Sigma(f)=\Sigma(g)$ be a cuspidal edge of $f$ (resp. $g$ ) and $\gamma$ a singular curve through $p=\gamma(0)$. Then $\gamma$ is a line of curvature of $f$ (resp. $g$ ) if and only if $\kappa_{t}^{h}$ (resp. $\left.\kappa_{t}^{d}\right)$ vanishes identically on $\gamma$.

Although this is shown for the case of fronts in Euclidean 3-space (see [34, Proposition 3.3], [15, p. 95]), and the same proof works, we give a proof here using the representation formula.

Proof. The curve $\gamma$ is a line of curvature of $f$ (resp. $g$ ) if and only if the following equation holds:

$$
\Omega(\xi f, g, \xi g)(\gamma(t))=0 \quad(\operatorname{resp} . \Omega(\xi g, f, \xi f)(\gamma(t))=0) .
$$

By a direct calculation, we see that

$$
\begin{aligned}
\Omega(\xi f, g, \xi g)(\gamma(t)) & =\langle\xi f \times g, \xi g\rangle(\gamma(t)) \\
& =4 \operatorname{Re}\left(\sqrt{\alpha \beta} \lambda_{\bar{z}}\right)(\gamma(t)) \operatorname{Im}\left(\sqrt{\alpha \beta} \lambda_{\bar{z}}\right)(\gamma(t)) \\
\text { (resp. } \Omega(\xi g, f, \xi f)(\gamma(t)) & =\langle\xi g \times f, \xi f\rangle(\gamma(t)) \\
& \left.=-4 \operatorname{Re}\left(\sqrt{\alpha \beta} \lambda_{\bar{z}}\right)(\gamma(t)) \operatorname{Im}\left(\sqrt{\alpha \beta} \lambda_{\bar{z}}\right)(\gamma(t))\right) .
\end{aligned}
$$

Thus we have the assertion by Theorem 3.4 if $f$ (resp. $g$ ) has cuspidal edges along $\gamma$.

We define another kind of singular point so called cone-like singularity ([20, p. $305])$.

Definition 4.2. Let $h: U \rightarrow M$ be a frontal from a region into a 3 -dimensional manifold. Let $p \in U$ be a non-degenerate singular point. Then $p$ is a cone-like singularity if there exists a neighborhood $V$ of $p$ such that for any $q \in \Sigma(h)$, a null vector field $\eta_{q}$ is tangent to $\Sigma(h)$.

Assume that the singular curve $\gamma$ of $f$ (resp. $g$ ) consists of cuspidal edges. By Proposition 4.1, if $\gamma$ is a line of curvature of $f$ (resp. $g$ ), then $\xi$ and $\eta_{d}$ (resp. $\xi$ and $\eta_{h}$ ) are parallel along $\gamma$. Thus we have the following corollary. 
Corollary 4.3. Let $f: U \rightarrow H^{3}$ and $g: U \rightarrow S_{1}^{3}$ be flat fronts and $p \in \Sigma(f)=\Sigma(g)$ a non-degenerate singular point. If the singular curve $\gamma$ passing through $p$ consists of cuspidal edges of $f$ (resp. $g$ ) and is a line of curvature of $f$ (resp. g), then $g$ (resp. f) has a cone-like singularity at $p$.

Acknowledgments. The authors would like to thank Masaaki Umehara for valuable comments, and thank Shun Iguchi and Mao Nomura for helping calculations.

\section{REFERENCES}

[1] D. Brander and F. Tari, Families of spherical surfaces and harmonic maps preprint, arXiv:1709.00984

[2] A. V. Corro, A. Martínez and K. Tenenblat, Ribaucour transformations for flat surfaces in the hyperbolic 3-space, J. Math. Anal. Appl. 412 (2014), no. 2, 720-743.

[3] J. M. Espinar, J. A. Gálvez and P. Mira, Hypersurfaces in $H^{n+1}$ and conformally invariant equations: the generalized Christoffel and Nirenberg problems, J. Eur. Math. Soc. 11 (2009), no. 4, 903-939.

[4] S. Fujimori, M. Noro, K. Saji, T. Sasaki and M. Yoshida, Schwarz maps for the hypergeometric differential equation, Internat. J. Math. 26 (2015), no. 6, 1541002, 31 pp.

[5] T. Fukui, Local differential geometry of cuspidal edge and swallowtail, preprint.

[6] J. A. Gálvez, A. Martínez and F. Milán, Flat surfaces in the hyperbolic 3-space, Math. Ann. 316 (2000), no. 3, 419-435.

[7] J. A. Gálvez, A. Martínez and F. Milán, Complete linear Weingarten surfaces of Bryant type. A Plateau problem at infinity, Trans. Amer. Math. Soc. 356 (2004), no. 9, 3405-3428.

[8] J. A. Gálvez and P. Mira, Embedded isolated singularities of flat surfaces in hyperbolic 3-space, Calc. Var. Partial Differential Equations 24 (2005), no. 2, 239-260.

[9] M. Hasegawa, A. Honda, K. Naokawa, K. Saji, M. Umehara and K. Yamada, Intrinsic properties of surfaces with singularities, Internat. J. Math. 26 (2015), no. 4, 1540008, 34 pp.

[10] A. Honda, K. Naokawa, M. Umehara and K. Yamada, Isometric deformations of wave fronts at non-degenerate singular points, preprint, arXiv:1710.02999

[11] S. Izumiya, Legendrian dualities and spacelike hypersurfaces in the lightcone, Mosc. Math. J. 9 (2009), no. 2, 325-357.

[12] S. Izumiya, M. C. Romero Fuster, M. A. S. Ruas and F. Tari, Differential geometry from a singularity theory viewpoint, World Scientific Publishing Co. Pte. Ltd., Hackensack, NJ, 2016.

[13] S. Izumiya and K. Saji, The mandala of Legendrian dualities for pseudo-spheres in LorentzMinkowski space and "flat" spacelike surfaces, J. Singul. 2 (2010), 92-127.

[14] S. Izumiya, K. Saji and M. Takahashi, Horospherical flat surfaces in hyperbolic 3-space, J. Math. Soc. Japan 62 (2010), no. 3, 789-849.

[15] S. Izumiya, K. Saji and N. Takeuchi, Flat surfaces along cuspidal edges, J. Singul. 16 (2017), 73-100.

[16] Y. Kitagawa and M. Umehara, Extrinsic diameter of immersed flat tori in $S^{3}$, Geom. Dedicata 155 (2011), 105-140.

[17] M. Kokubu, Surfaces and fronts with harmonic-mean curvature one in hyperbolic three-space, Tokyo J. Math. 32 (2009), no. 1, 177-200.

[18] M. Kokubu, W. Rossman, M. Umehara and K. Yamada, Flat fronts in hyperbolic 3-space and their caustics, J. Math. Soc. Japan 59 (2007), no. 1, 265-299.

[19] M. Kokubu, W. Rossman, M. Umehara and K. Yamada, Asymptotic behavior of flat surfaces in hyperbolic 3-space, J. Math. Soc. Japan 61 (2009), no. 3, 799-852.

[20] M. Kokubu, W. Rossman, K. Saji, M. Umehara and K. Yamada, Singularities of flat fronts in hyperbolic space, Pacific J. Math. 221 (2005), no. 2, 303-351.

[21] M. Kokubu, M. Umehara and K. Yamada, Flat fronts in hyperbolic space, Pacific J. Math. 216 (2004), no. 1, 149-175.

[22] M. Kokubu and M. Umehara, Orientability of linear Weingarten surfaces, spacelike CMC-1 surfaces and maximal surfaces, Math. Nachr. 284 (2011), no. 14-15, 1903-1918.

[23] H. Liu and S. D. Jung, Hypersurfaces in lightlike cone, J. Geom. Phys. 58 (2008), no. 7, 913-922.

[24] H. Liu, M. Umehara and K. Yamada, The duality of conformally flat manifolds, Bull. Braz. Math. Soc. 42 (2011), no. 1, 131-152. 
[25] A. Martínez and F. Milán, Flat fronts in hyperbolic 3-space with prescribed singularities, Ann. Global Anal. Geom. 46 (2014), no. 3, 227-239.

[26] L. F. Martins and K. Saji, Geometric invariants of cuspidal edges, Canad. J. Math. 68 (2016), no. $2,445-462$.

[27] L. F. Martins, K. Saji, M. Umehara and K. Yamada, Behavior of Gaussian curvature and mean curvature near non-degenerate singular points on wave fronts, Geometry and Topology of Manifold, Springer Proc. Math. \& Statistics, 2016, 247-282.

[28] R. Oset Sinha and F. Tari, Flat geometry of cuspidal edges, to appear in Osaka J. Math. arXiv: 1610.08702

[29] K. Saji, M. Umehara, and K. Yamada, The geometry of fronts, Ann. of Math. 169 (2009), 491-529.

[30] K. Saji, M. Umehara and K. Yamada, $A_{k}$ singularities of wave fronts, Math. Proc. Cambridge Philos. Soc. 146 (2009), no. 3, 731-746.

[31] T. Sasaki, K. Yamada and M. Yoshida, The hyperbolic Schwarz map for the hypergeometric differential equation, Experiment. Math. 17 (2008), no. 3, 269-282.

[32] T. Sasaki, K. Yamada and M. Yoshida, Derived Schwarz map of the hypergeometric differential equation and a parallel family of flat fronts, Internat. J. Math. 19 (2008), no. 7, 847-863.

[33] K. Teramoto, Parallel and dual surfaces of cuspidal edges, Differential Geom. Appl. 44 (2016), $52-62$.

[34] K. Teramoto, Principal curvatures and parallel surfaces of wave fronts, to appear in Adv. Geom., arXiv:1612.00577

[35] K. Teramoto, Focal surfaces of wave fronts in the Euclidean 3-space, to appear in Glasgow Math. J., arXiv:1804.06123

(K. Saji) Department of Mathematics, Graduate School of Science, Kobe University, ROKKODAi 1-1, NADA, KOBE 657-8501, JAPAN

E-mail address: sajilmath.kobe-u.ac.jp

(K. Teramoto) Department of Mathematics, Graduate School of Science, Kobe UniVERsity, RokKodai 1-1, NADA, Kobe 657-8501, JAPAN

E-mail address: teramotolmath.kobe-u.ac.jp 